\title{
Locating Areas of High Density of Plant Parasitic Nematode, Rotylenchulus spp. in Vidarbha Region of Maharashtra State, India
}

\author{
N. V. Lavhe ${ }^{1 *}$, D. B. Undirwade ${ }^{2}$, Nandini Gokte- Narkhedkar ${ }^{3}$ and S. L. Borkar ${ }^{1}$ \\ ${ }^{1}$ Department of Entomology, College of Agriculture, Nagpur, Dr. PDKV, Akola, India \\ ${ }^{2}$ ICAR-CICR, Nagpur, India \\ *Corresponding author
}

\section{A B S T R A C T}

\section{Keywords}

Plant parasitic nematodes, Genera, Rotylenchulus spp., High density spots

Article Info

Accepted:

04 November 2019

Available Online:

10 December 2019
Vidarbha region of Maharashtra comprising of two divisions i.e. Amravati and Nagpur was surveyed for locating high density spots of plant parasitic nematodes, Rotylenchulus spp.. About 737 soil samples were collected from 16 crop systems in 119 talukas and evaluated for presence of plant parasitic nematodes. In literature, amongst the genera of plant parasitic nematodes, threshold levels of damage have been standardised for only 5 genera. Mapping of plant parasitic nematodes was done crop ecosystem wise. Reniform nematode, Rotylenchulus reniformis was the most prevalent and predominant nematode species. This may be attributed to the fact that major crops of the region viz. Cotton, Soybean and pigeon pea are also good hosts for this nematode. The areas where the population exceeds the threshold of damage (200/100cc soil) for reniform nematode $R$. Reniformis were identified and mapped. Maximum number of samples from Cotton crop showing high population density of reniform nematode was recorded in Yavatmal district followed by Buldhana while population was less in Nagpur and Gadchiroli. For Pigeon pea crop also high density spots were recorded highest in Yavatmal followed by Buldhana and Amravati with lowest in Gadchiroli. Variation in population density may probably be correlated to gradual spread of irrigation availability through drip irrigation.

\section{Introduction}

There are numerous estimates of the economic importance of nematodes in crop production on a world wide and individual country basis, but precise estimation of losses is lacking. Nematodes are of major importance to agricultural crops as environment is congenial for nematode population build up. Importance of nematodes as disease causing entities have often been overlooked. Symptoms caused due to nematode infestation are non-specific and mistaken for nutritional deficiency ones. Nematode damage to crops is dependent on nematode density in soil. Damage caused by nematodes gets manifested only when 
nematode populations cross threshold level of economic damage.

In areas as Buldhana where cotton is increasingly being grown under drip under intensive cultivation, reniform nematode infestation is being reported (Agro one, 2 Nov, 2016). Root-knot nematode ( $M$. incognita) is emerging as major problem in pomegranate growing areas in and around Rahuri. Similarly, burrowing nematodes ( $R$. similis) is also being reported from banana growing areas. Survey on various crops grown in polyhouses has reported severe infestation of species like $M$. incognita, $R$. reniformis and Xiphinema sp. In Pune, Satara, Sangli and Kolhapur districts.(Anonymous, 2010). There is a general lack of awareness of nematodes due to its microscopic size and non-specific symptoms. Symptoms of nematode damage are non-specific and resemble those of nutrient deficiency. Furthermore nematode distribution and damage within a field occurs in patches. Nematode damaged crops infestation appears as irregular patches or streaks that may vary in size, shape, and number. These variations usually reflect the compounding of nematode stress on a plant by such other factors as nutritional denomolies, physical soil differences and irrigation and drainage patterns. Spread of nematodes is aided by irrigation water. Dozens of different genera of plant parasitic nematodes have been reported to spread through irrigation canals. Nematode damage is density dependent. Damage due to nematodes gets manifested only when population crosses damage threshold which vary with nematode species as well as host crop. To create awareness about plant parasitic nematodes, diagnosis of symptoms on plants is first step towards ameliorating losses caused due to nematodes.

Nematode problems are more severe in tropics and horticultural crops, annuals under irrigation and in protected cultivation.
Nematode damage is density dependent and gets manifested when population exceeds threshold level of damage.

\section{Materials and Methods}

The research work was carried out to Locate areas of High density of plant parasitic nematode, Rotylenchulus spp. in Vidarbha region". The survey was conducted for two divisions i.e. Amravati and Nagpur comprising of 119 talukas covering about $9762 \mathrm{kms}$. The soil samples were collected from each taluka and evaluated for the high density spots of nematode population were determined.

\section{Experimental details}

The soil samples were collected from different cropping systems at different locations in the Vidrabha region.

The Samples were collected from different agroecosystems i.e. cotton, pigeon pea, paddy, vegetables, sugarcane, citrus, forest trees, pomegranate, beetle vine, chilli, turmeric, banana etc. which represent the crop ecosystem. These samples were brought to the laboratory and further extraction of nematodes was done by soil sieving and decanting technique developed by Dr. N.A.Cobb (1918) in the Nematology Laboratory, Crop Protection Division, CICR, Nagpur.

\section{Statistical analysis}

Shannon Evenness Index $(E)=H / L n ~ S$

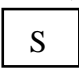

Shannon Diversity $\operatorname{Index}(H)=-\sum$ pi $\ln$ pi

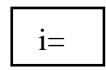

The population densities of nematode species in the samples were calculated by the formulae given by Norton 1978 . 


\section{Morphological characterization}

Nematodes were killed and fixed by adding equal amount of hot fixative formaldehyde acetic acid (FAA).

Morphometric studies were undertaken for identification and characterisation of nematodes.

\section{Results and Discussion}

The survey was conducted for two divisions i.e. Amravati and Nagpur comprising of 119 talukas covering about $9762 \mathrm{kms}$. The soil samples were collected from each taluka and evaluated for presence of plant parasitic nematodes and high density spots were determined by mapping the nematodes qualitatively and quantitatively crop ecosystem wise.

\section{Experimental Findings}

The present investigations for Locating areas of High density of plant parasitic nematode, Rotylenchulus spp. were carried out from all 11 districts and 119 talukas of Vidarbha region. In about 16 crops plant parasitic nematode Rotylenchulus spp. were reported. This was the first ever work done in Vidarbha region.

Amongst all the genera Rotylenchulus, Hoplolaimus, Helicotylenchus, Pratylenchus, Meloidogyne, Tylenchulus, Paratylenchus was prominently recorded in all the districts.

Crop ecosystem wise mapping of plant parasitic nematodes on the base of prevalence and distribution

On the basis of prevalence and distribution, mapping of plant parasitic nematodes was done crop ecosystem wise. From the mapping the high density spots were identified based on the nematode densities with populations exceeding the damage threshold to point out the possible problem areas with potential damage in Table 1 to 5 .

Genera wise high density spots of plant parasitic nematodes

Amongst genera of plant parasitic nematodes isolated and identified. The genera Rotylenchulus, Hoplolaimus, Helicotylenchus, Pratylenchus, Meloidogyne, Tylenchulus, Paratylenchus was prominently recorded in all the districts. According to the damage threshold of the nematodes the high density spots were identified. The damage thresholds for genera Rotylenchulus (200/100cc soil) (Gokte-Narkhedkar et al., 2004).

From this it was evident that genus Rotylenchulus spp has recorded the high density population on 170 spots in 119 talukas comprising of 39 crop ecosystems of Vidarbha region.

\section{Crop wise high density spots}

\section{Cotton}

It was revealed that high density spots for cotton crop for genera Rotylenchulus were 59 from 9 districts with 3 talukas from Nagpur, 12 talukas from Buldhana, 5 talukas from Wardha,04 talukas from Washim, 16 talukas from Yavatmal, 07 talukas from Chandrapur, 3 talukas from Gadchiroli, 5 talukas from Akola and 4 talukas from Amravati.

\section{Pigeonpea}

High density spots identified for Pigeon pea crop for genera Rotylenchulus were 75 from 11 districts with 14 talukas from Buldhana district, 5 talukas each from Wardha and Akola,4 talukas each from Gondia and Washim, 12 talukas from Amravati, 15 talukas 
from Yavatmal, 3 talukas from Nagpur, 7 talukas from Chandrapur and 2 talukas from Bhandara and Gadchiroli each.

\section{Citrus}

High density spots where the population has exceeded the threshold level for citrus crop for genus Rotylenchulus were at 2 locations in 2 districts 1 taluka each from Wardha and Nagpur.

\section{Brinjal}

Genera Rotylenchulus recorded 4 spots where the population has exceeded the threshold level from 4 districts with 1 taluka each from Gadchiroli, Gondia, Bhandara and Nagpur.

\section{Soybean}

It was revealed that high density spots were identified for soybean crop for genera Rotylenchulus were 23 from 6 districts with 10 talukas from Buldhana, 6 talukas from Washim, 3 talukas from akola, 2 talukas from Wardha and 1 taluka each from Amravati and Nagpur respectively.

\section{Chilli/ Wal/ Tomato/ Onion/ Cowpea/ Turmeric}

High density spots identified for Chilli crop was Korpana, for Wal crop were Narkhed for genera Rotylenchulus. In Tomato crop high density spots were recorded where the population has exceeded the threshold level were in 3 districts with 1 taluka each from Gadchiroli, Wardha and Gondia for genera Rotylenchulus. While Onion crop was identified with 1 high density spot each in Nagpur district for genera Rotylenchulus. High density spots where the population has exceeded the threshold level were recorded for crop Cowpea were from 3 districts with 1 taluka each from Gadchiroli, Chandrapur and Gondia for genera Rotylenchulus. However high density spots with exceeded threshold level were identified for genera Roylenchulus from 1 taluka each of Yavatmal Gadchiroli and Gondia district for Turmeric and Bendi crop.

\section{Bhendi/ Cucumber/ Gerbera/ Radish}

High density spots for Bhendi where the population has exceeded the threshold level were identified for genera Rotylenchulus were 1taluka each from Gadchiroli and Gondia,

In Cucumber crop Gadchiroli district has identified 1 taluka as high density spot for genera Rotylenchulus, high density spots where the population has exceeded the threshold level were identified for Gerbera crop for genera Rotylenchus, 1 taluka from Gondia district.

Radish crop recorded high density spots for genera Rotylenchulus, in 1 taluka of Gadchiroli district.

Table.1 High density spots of plant parasitic nematode Rotylenchulus spp. in Vidarbha region

\begin{tabular}{|c|c|c|c|c|c|c|c|c|c|c|c|}
\hline \multirow[t]{2}{*}{ 莺 } & \multirow[t]{2}{*}{ 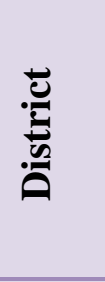 } & 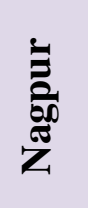 & 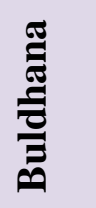 & 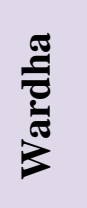 & 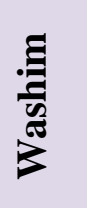 & 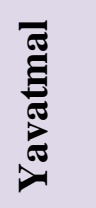 & 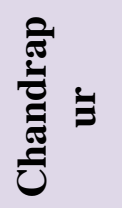 & 音 & $\frac{\pi}{\frac{\pi}{0}}$ & 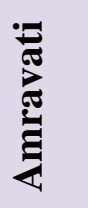 & تِّتٍ: \\
\hline & & 1 & 2 & 3 & 4 & 5 & 6 & 7 & 8 & 9 & 10 \\
\hline \multicolumn{2}{|c|}{ Rotylen chulus } & 13 & 36 & 14 & 14 & 32 & 16 & 11 & 13 & 17 & 8 \\
\hline
\end{tabular}


Table.2 High density spots of plant parasitic nematodes Rotylenchulus spp. in Districts (No. of talukas)

\begin{tabular}{|c|c|c|c|}
\hline S.No. & Crop & District & No. of talukas \\
\hline \multirow[t]{9}{*}{1} & Cotton & Nagpur & 3 \\
\hline & & Buldhana & 12 \\
\hline & & Wardha & 5 \\
\hline & & Washim & 4 \\
\hline & & Yavatmal & 16 \\
\hline & & Chandrapur & 7 \\
\hline & & Gadchiroli & 3 \\
\hline & & Akola & 5 \\
\hline & & Amravati & 4 \\
\hline \multirow[t]{11}{*}{2} & Pigeonpea & Nagpur & 5 \\
\hline & & Buldhana & 14 \\
\hline & & Wardha & 5 \\
\hline & & Washim & 4 \\
\hline & & Yavatmal & 15 \\
\hline & & Chandrapur & 7 \\
\hline & & Gadchiroli & 2 \\
\hline & & Akola & 5 \\
\hline & & Amravati & 12 \\
\hline & & Gondia & 4 \\
\hline & & Bhandara & 2 \\
\hline \multirow[t]{2}{*}{3} & Citrus & Nagpur & 1 \\
\hline & & Wardha & 1 \\
\hline \multirow[t]{4}{*}{4} & Brinjal & Nagpur & 1 \\
\hline & & Gadchiroli & 1 \\
\hline & & Gondia & 1 \\
\hline & & Bhandara & 1 \\
\hline \multirow[t]{6}{*}{5} & Soybean & Nagpur & 1 \\
\hline & & Buldhana & 10 \\
\hline & & Wardha & 2 \\
\hline & & Washim & 6 \\
\hline & & Akola & 3 \\
\hline & & Amravati & 1 \\
\hline 6 & Chilli & Chandrapur & 1 \\
\hline 7 & Wal(Dolichos) & Nagpur & 1 \\
\hline \multirow[t]{3}{*}{8} & Tomato & Wardha & 1 \\
\hline & & Gadciroli & 1 \\
\hline & & Gondia & 1 \\
\hline 10 & Onion & Nagpur & 1 \\
\hline \multirow[t]{3}{*}{11} & Cowpea & Chandrapur & 1 \\
\hline & & Gadciroli & 1 \\
\hline & & Gondia & 1 \\
\hline 12 & Turmeric & Yavatmal & 1 \\
\hline \multirow[t]{2}{*}{13} & Bhendi & Gadchiroli & 1 \\
\hline & & Gondia & 1 \\
\hline 14 & Cucumber & Gadchiroli & 1 \\
\hline 15 & Gerbera & Gondia & 1 \\
\hline 16 & Radish & Gadchiroli & 1 \\
\hline
\end{tabular}


Table.3 Talukawise average population of plant parasitic nematode Rotylenchulus spp. in 100gm soil in Cotton crop in Vidarbha region

\begin{tabular}{|c|c|c|c|c|c|c|c|c|c|c|c|c|c|}
\hline \multirow[t]{2}{*}{$\begin{array}{l}\frac{0}{\pi} \\
0 \\
0 \\
0\end{array}$} & \multirow[t]{2}{*}{ 胥 } & 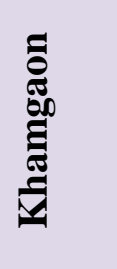 & 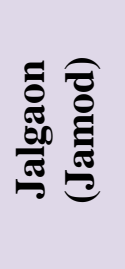 & 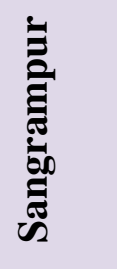 & 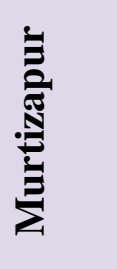 & 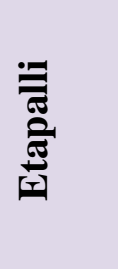 &  & 苛 & $\frac{2}{2}$ & 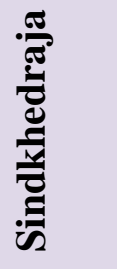 & 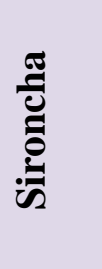 & 㐫 & 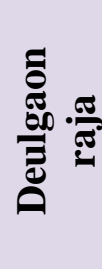 \\
\hline & & 1 & 2 & 3 & 4 & 5 & 6 & 7 & 8 & 9 & 10 & 11 & 12 \\
\hline \multicolumn{2}{|c|}{ Rotylenchulus } & 470 & 380 & 367 & 360 & 340 & 320 & 320 & 320 & 310 & 300 & 300 & 300 \\
\hline
\end{tabular}

\begin{tabular}{|c|c|c|c|c|c|c|c|c|c|c|c|c|c|}
\hline \multirow[t]{2}{*}{$\begin{array}{c}\text { Isola } \\
\text { te }\end{array}$} & \multirow[t]{2}{*}{$\begin{array}{c}\text { Talu } \\
\text { ka }\end{array}$} & $\begin{array}{l}\text { Chand } \\
\text { ur Rly }\end{array}$ & $\begin{array}{c}\text { Umarkh } \\
\text { ed }\end{array}$ & $\begin{array}{c}\text { Jiva } \\
\text { ti }\end{array}$ & $\begin{array}{c}\text { War } \\
\text { ud }\end{array}$ & $\begin{array}{c}\text { Marega } \\
\text { on }\end{array}$ & $\begin{array}{c}\text { Mano } \\
\text { ra }\end{array}$ & $\begin{array}{c}\text { Malkap } \\
\text { ur }\end{array}$ & $\begin{array}{c}\text { Korpa } \\
\text { na }\end{array}$ & $\begin{array}{c}\text { Karanja(La } \\
\text { ad) }\end{array}$ & $\begin{array}{c}\text { Waro } \\
\text { ra }\end{array}$ & $\begin{array}{l}\text { Mehk } \\
\text { ar }\end{array}$ & $\begin{array}{c}\text { Kelap } \\
\text { ur }\end{array}$ \\
\hline & & 13 & 14 & 15 & 16 & 17 & 18 & 19 & 20 & 21 & 22 & 23 & 24 \\
\hline \multicolumn{2}{|c|}{ Rotylenchulus } & 300 & 290 & 288 & 280 & 280 & 280 & 280 & 280 & 280 & 272 & 270 & 270 \\
\hline
\end{tabular}

\begin{tabular}{|c|c|c|c|c|c|c|c|c|c|c|c|c|c|}
\hline \multirow[t]{2}{*}{$\begin{array}{c}\text { Isola } \\
\text { te }\end{array}$} & \multirow[t]{2}{*}{$\begin{array}{c}\text { Talu } \\
\text { ka }\end{array}$} & $\begin{array}{c}\text { Kala } \\
\text { mb }\end{array}$ & $\begin{array}{c}\text { Ghata } \\
\text { nji }\end{array}$ & $\begin{array}{c}\text { Mahaga } \\
\text { on }\end{array}$ & $\begin{array}{c}\text { Deo } \\
\text { li }\end{array}$ & $\begin{array}{c}\text { Yavat3 } \\
\text { mal }\end{array}$ & $\begin{array}{c}\text { Telha } \\
\text { ra }\end{array}$ & $\begin{array}{c}\text { Pandharkaw } \\
\text { ada }\end{array}$ & $\begin{array}{l}\text { Narkh } \\
\text { ed }\end{array}$ & $\begin{array}{l}\text { Nandu } \\
\text { ra }\end{array}$ & $\begin{array}{l}\text { Mota } \\
\text { la }\end{array}$ & $\begin{array}{c}\text { Dhamanga } \\
\text { on RLy }\end{array}$ & $\begin{array}{c}\text { Chim } \\
\text { ur }\end{array}$ \\
\hline & & 25 & 26 & 27 & 28 & 29 & 30 & 31 & 32 & 33 & 34 & 35 & 36 \\
\hline \multicolumn{2}{|c|}{ Rotylenchulus } & 270 & 270 & 267 & 260 & 260 & 260 & 260 & 260 & 260 & 260 & 260 & 260 \\
\hline
\end{tabular}

\begin{tabular}{|c|c|c|c|c|c|c|c|c|c|c|c|c|c|}
\hline \multirow[t]{2}{*}{ Isolate } & \multirow[t]{2}{*}{ Taluka } & Chikhali & Ashti & Zarijamani & Patur & Malegaon & Ralegaon & Wardha & Samudrapur & Arni & Wani & Mangrulpir & Chandrapur \\
\hline & & 37 & 38 & 39 & 40 & 41 & 42 & 43 & 44 & 45 & 46 & 47 & 48 \\
\hline \multicolumn{2}{|c|}{ Rotylenchulus } & 260 & 253 & 250 & 250 & 250 & 248 & 247 & 247 & 247 & 240 & 240 & 240 \\
\hline
\end{tabular}

\begin{tabular}{|c|c|c|c|c|c|c|c|c|c|c|c|c|}
\hline \multirow[t]{2}{*}{ Isolate } & \multirow[t]{2}{*}{ Taluka } & Barshitakli & Arvi & Rajura & Risod & Katol & Parseoni & Buldhana & Tiwasa & $\begin{array}{c}\text { Balapur } \\
\text { (Wadegaon) }\end{array}$ & Babhulgaon & Darwha \\
\hline & & 49 & 50 & 51 & 52 & 53 & 54 & 55 & 56 & 57 & 58 & 59 \\
\hline \multicolumn{2}{|c|}{ Rotylenchulus } & 240 & 240 & 233 & 230 & 230 & 220 & 220 & 210 & 210 & 210 & 200 \\
\hline
\end{tabular}


Table.4 Talukawise average population of plant parasitic nematode Rotylenchulus spp. per 100gm soil in Pigeonpea crop in Vidarbha region

\begin{tabular}{|c|c|c|c|c|c|c|c|c|c|c|c|c|c|}
\hline \multirow[t]{2}{*}{ Isolate } & \multirow[t]{2}{*}{ Taluka } & Khamgaon & Deori & Tirora & Shegaon & Jalgaon(Jamod) & $\begin{array}{c}\text { Balapur } \\
\text { (Wadegaon) }\end{array}$ & $\begin{array}{c}\text { Dhamangaon } \\
\text { RLy }\end{array}$ & Sangrampur & Motala & Warud & Dharni & Achalpur \\
\hline & & 1 & 2 & 3 & 4 & 5 & 6 & 7 & 8 & 9 & 10 & 11 & 12 \\
\hline \multicolumn{2}{|c|}{ Rotylenchulus } & 390 & 380 & 370 & 370 & 360 & 360 & 340 & 330 & 320 & 320 & 313 & 310 \\
\hline
\end{tabular}

\begin{tabular}{|c|c|c|c|c|c|c|c|c|c|c|c|c|c|c|c|c|c|}
\hline \multirow[t]{2}{*}{ Isolate } & \multirow[t]{2}{*}{ Taluka } & \multicolumn{2}{|c|}{ Chikhaldara } & \multicolumn{2}{|c|}{ Korpana } & Ralegaon & \multicolumn{2}{|c|}{ Mahagaon } & Umarkhed & Bhatkuli & Daryapur & Warora & Manora & $\begin{array}{c}\text { Nandgaon } \\
\text { Khandeshwar }\end{array}$ & \multicolumn{2}{|c|}{ Gondpipri } & Ner \\
\hline & & \multicolumn{2}{|c|}{13} & \multicolumn{2}{|c|}{14} & 15 & \multicolumn{2}{|l|}{16} & 17 & 18 & 19 & 20 & 21 & 22 & \multicolumn{2}{|r|}{23} & 24 \\
\hline \multicolumn{2}{|c|}{ Rotylenchulus } & \multicolumn{2}{|c|}{308} & \multicolumn{2}{|c|}{300} & 300 & \multicolumn{2}{|l|}{300} & 300 & 300 & 296 & 290 & 290 & 290 & & 280 & 280 \\
\hline \multirow[t]{2}{*}{ Isolate } & \multirow[t]{2}{*}{ Taluka } & Lonar & \multicolumn{2}{|c|}{ Nagbhid } & \multicolumn{2}{|c|}{ Mangrulpir } & Malkapur & Wani & Arvi & Risod & Mehkar & Darwha & Kelapur & \multicolumn{2}{|c|}{ Chandurbazaar } & \multicolumn{2}{|c|}{ Tiwasa } \\
\hline & & 25 & \multicolumn{2}{|c|}{26} & \multicolumn{2}{|r|}{27} & 28 & 29 & 30 & 31 & 32 & 33 & 34 & \multicolumn{2}{|l|}{35} & \multicolumn{2}{|c|}{36} \\
\hline \multicolumn{2}{|c|}{ Rotylenchulus } & 275 & \multicolumn{2}{|c|}{270} & \multicolumn{2}{|r|}{270} & 270 & 270 & 260 & 260 & 260 & 260 & 260 & \multicolumn{2}{|l|}{260} & 26 & \\
\hline
\end{tabular}

\begin{tabular}{|c|c|c|c|c|c|c|c|c|c|c|c|c|c|}
\hline \multirow[t]{2}{*}{ Isolate } & Taluka & Amravati & Gadchiroli & Zarijamani & Patur & Murtizapur & Telhara & Sakoli & Maregaon & Ramtek & Wardha & M.Arjuni & Gondia \\
\hline & & 37 & 38 & 39 & 40 & 41 & 42 & 43 & 44 & 45 & 46 & 47 & 48 \\
\hline \multicolumn{2}{|c|}{ Rotylenchulus } & 260 & 250 & 250 & 250 & 250 & 250 & 247 & 247 & 240 & 240 & 240 & 240 \\
\hline
\end{tabular}

\begin{tabular}{|c|c|c|c|c|c|c|c|c|c|c|c|c|c|}
\hline \multirow[t]{2}{*}{ Isolate } & \multirow[t]{2}{*}{ Taluka } & Rajura & Chimur & Malegaon & $\begin{array}{c}\text { Karanja } \\
\text { (Laad) }\end{array}$ & $\begin{array}{c}\text { Deulgaon } \\
\text { raja }\end{array}$ & Chikhali & Sindkhedraja & Ghatanji & Arni & Ashti & Chamorshi & Pusad \\
\hline & & 49 & 50 & 51 & 52 & 53 & 54 & 55 & 56 & 57 & 58 & 59 & 60 \\
\hline \multicolumn{2}{|c|}{ Rotylenchulus } & 240 & 240 & 240 & 240 & 240 & 240 & 240 & 240 & 240 & 233 & 230 & 230 \\
\hline
\end{tabular}

\begin{tabular}{|c|c|c|c|c|c|c|c|c|c|c|c|c|c|c|}
\hline \multirow[t]{2}{*}{ Isolate } & \multirow[t]{2}{*}{ Taluka } & Buldhana & Parseoni & Samudrapur & $\begin{array}{l}\text { Sadak } \\
\text { arjuni }\end{array}$ & Mul & Kalamb & Barshitakli & Tumsar & Nandura & Narkhed & Babhulgaon & Digras & $\begin{array}{c}\text { Chandur } \\
\text { rly }\end{array}$ \\
\hline & & 61 & 62 & 63 & 64 & 65 & 66 & 67 & 68 & 69 & 70 & 71 & 72 & 73 \\
\hline \multicolumn{2}{|c|}{ Rotylenchulus } & 227 & 220 & 220 & 220 & 220 & 220 & 220 & 210 & 210 & 200 & 200 & 200 & 200 \\
\hline
\end{tabular}


Table.5 Talukawise average population of plant parasitic nematode Rotylenchulus spp. per $100 \mathrm{gm}$ soil in various crops in Vidarbha region

\begin{tabular}{|c|c|c|c|}
\hline S.no. & Crop & Taluka & $\begin{array}{l}\text { Damage threshold } \\
\text { (200/100cc soil) }\end{array}$ \\
\hline \multirow[t]{2}{*}{1} & Citrus & Ashti & 240 \\
\hline & & Kalmeshwar & 230 \\
\hline \multirow[t]{4}{*}{2} & Brinjal & Hingna & 220 \\
\hline & & Armori & 220 \\
\hline & & M.arjuni & 200 \\
\hline & & Sindewahi & 200 \\
\hline \multirow[t]{23}{*}{3} & Soybean & Amravati & 370 \\
\hline & & Jalgaon(Jamod) & 360 \\
\hline & & Balapur(wadegaon) & 340 \\
\hline & & Mangrulpir & 300 \\
\hline & & Motala & 300 \\
\hline & & Buldhana & 300 \\
\hline & & Sindkhedraja & 280 \\
\hline & & Malkapur & 260 \\
\hline & & Mehkar & 260 \\
\hline & & Khamgaon & 250 \\
\hline & & Karanja & 240 \\
\hline & & Ashti & 240 \\
\hline & & Karanja(Laad) & 240 \\
\hline & & Chikhali & 240 \\
\hline & & Lonar & 240 \\
\hline & & Barshitakli & 240 \\
\hline & & Shegaon & 230 \\
\hline & & Patur & 220 \\
\hline & & Washim & 210 \\
\hline & & Deulgaonraja & 210 \\
\hline & & Katol & 200 \\
\hline & & Risod & 200 \\
\hline & & Malegaon & 200 \\
\hline 4 & Chilli & Korpana & 200 \\
\hline 5 & Wal (Dolichos) & Narkhed & 220 \\
\hline \multirow[t]{3}{*}{6} & Tomato & Etapalli & 270 \\
\hline & & Samudrapur & 240 \\
\hline & & Morgaon arjuni & 240 \\
\hline 7 & Onion & Hingna & 210 \\
\hline \multirow[t]{3}{*}{8} & Cowpea & Gadchiroli & 340 \\
\hline & & Bhadravati & 260 \\
\hline & & Goregaon & 200 \\
\hline 9 & Turmeric & Mahagaon & 200 \\
\hline \multirow[t]{2}{*}{10} & Bhendi & Gadchiroli & 260 \\
\hline & & Gondia & 200 \\
\hline 11 & Cucumber & Gadchiroli & 270 \\
\hline 12 & Gerbera & Gondia & 200 \\
\hline 13 & Radish & Mulchera & 200 \\
\hline
\end{tabular}


On the basis of prevalence and distribution, mapping of plant parasitic nematodes was done crop ecosystem wise. From the mapping the spots were identified based on the nematode densities with populations exceeding the damage threshold to point out the possible problem areas. The damage thresholds for genera Rotylenchulus (200/100cc soil) (Gokte-Narkhedkar et al., 2004).

Rotylenchulus reniformis recorded high frequency and density in all the 11 districts, 119 talukas and 39 crops of Vidarbha region.

Major crops infested by Rotylenchulus are brinjal, cabbage, chilli, cotton, citrus, pigeon pea, soybean, okra, pomogranate, radish, beetle vine, turmeric etc. Rotylenchulus is a polyphagus pest which has very wide host range.

Along with major infestation it also prevails with other forest and plantation crops also. Cotton is a very good and preferred host crop of this nematode (Gokte-Narkhedkar et al., 2006).

The high density spots were determined by mapping the nematodes crop ecosystem wise. On the basis of prevalence and distribution, mapping of plant parasitic nematodes was done crop ecosystem wise.

Most predominant nematode genera was Rotylenchulus and based on the population density hot spot areas for Cotton crop were recorded highest in Yavatmal followed by Buldhana with lowest in Nagpur and Gadchiroli.

Most predominant nematode genera was Rotylenchulus and based on the population density hot spot areas for Pigeon pea crop were recorded highest in Yavatmal followed by Buldhana and Amravati with lowest in
Gadchiroli and Bhandara. However Genera Rotylenchulus has shown predominance in Buldhana and Washim districts with 10 and 6 high density spots respectively in Soybean crop. Out of 19 genera, only five of the nematode genera has defined damage threshold levels and other genera so far not having defined level of damage threshold due to limited research work around the globe. The areas where the populations exceeding the damage threshold of nematode species Rotylenchulus spp. were also pointed out for genera Rotylenchulus (200/100cc soil) which constitute all crop ecosystems and districts of Vidarbha region.

\section{References}

Anonymous. 2011. Land Resources Atlas Vidarbha region. National Bureau of Soil Survey and Land Use Planning (NBSS\&LUP). NBSS\&LUP Publ.147. Pp-143.

Anonymous. 2010. Division of Nematology, Indian Agricultural Research Institute, NEW DELHI 110012

Anonymous. 2012. All India Co-ordinated Research project on plant parasitic nematodes at Department of Entomology, Mahatma Phule Krishi Vidyapeeth, Rahuri.

Deshmukh, Swati., Sunita Borde and Vishnu Barote. 2016. Prevalence of citrus nematodes in different localities around Aurangabad city, district Aurangabad (M.S.), India. Trends in Life sciences. Volume- 5 Issue- 2 (2016) ISSN: 23194731 (p); 2319-5037.

Gokte-Narkhedkar, N., Mukewar, P.M. and Mayee, C.D. 2004. Plant parasitic nematodes of cotton- Farmer's hidden enemy. Technical bulletin no.27, Central Institute for Cotton Research, Nagpur, $30 \mathrm{P}$.

Gokte-Narkhedkar, N., and Naikwade, A. 2016. Sutrakruminche Kapsi pikavaril Niyantran. Agro One Newspaper. 2 Nov, 2016, 16P. 
Kavitha and Vanita Das. 2015. Characterization of Plant-Parasitic Nematode Communities Associated With Brinjal Crops in the Nagole Area of Rangareddy District, Hyderabad, Telangana, India (2011-2012). Volume 5 Issue 5, May 2016. Int. J. of Science and Research (IJSR). 2292-2297.

Kayani, M. Z. T. Mukhtar, M. A. Hussain, M. I. Haque and R. Perveen. 2012. Incidence and severity of root-knot nematodes (Meloidogyne spp.) on Cucumber in District Rawalpindi. Pak. J. Phytopathol., Vol. 24(2):122-128, 2012.

Khan, M.R., R.K.Jain, T.M.Ghule and S. Pal. 2014. Root Knot Nematodes in India - A Comprehensive Monograph All India Coordinated Research Project on Plant Parasitic Nematodes with Integrated Approach for their Control Division of Nematology Indian Agricultural Research Institute New Delhi - 110012. PP-76

Khan.M.R., R.K.Jain. R.V.Singh and A.Pramanik. 2010. Maharashtra: Emerging Nematode problem. Economically Important Plant Parasitic Nematodes Distribution ATLAS. Directorate of Information and Publications of Agriculture Krishi Anusandhan Bhavan 1, Pusa New Delhi 110 012.Pp-105.

Kumar and Das. 2019. Diversity and community analysis of plant parasitic nematodes associated with citrus at citrus research station, Tinsukia, Assam. Journal of Entomology and Zoology Studies 2019; 7(3): 187-189

Lokesh Zalpuri, J.S. Tara, V.K. Singh. 2013. Prevalence of Plant Parasitic Nematodes (Citrus Species) in Various Villages of Jammu Region Int. J. of Scientific and Research Publications, Volume 3, Issue 6, June 20131 ISSN 2250-3153

Rashid Aasia, Shamim Ahmed Azad and Umar Farooq. 2014. Community analysis of plant parasitic nematodes associated with ornamental plants in Rajouri district (J\&K), India. Int.J.Curr.Microbiol. App.Sci (2014) 3(1): 194-197.

Sahu R., P. Chandra and A.N.Poddar. 2011. Community analysis of plant parasitic nematodes prevalent in vegetable crops in district Durg of Chattisgarh, India. Res. J. of Parasitology 6(2):83-89.2011.

Srinivasan, R, S. Kulothungan, P. Sundararaju and C. Govindasamy. 2011. Biodiversity of Plant Parasitic Nematodes associated with Banana in Thanjavur District of Tamilnadu. International Journal of Plant, Animal and Environmental Sciences. Volume-1, Issue-2 JuneAug:2011. Pp.63-69.

Srivastava, D.S., Mukesh Sehgal, Ajay Kumar, Savita Verma, B.K. Dwivedi And S.P. Singh. 2012. Incidence of Root-Knot Nematode Associated with Okra in District Allahabad, Uttar Pradesh, India. Indian Journal of Nematology. Vol. 42, No. 1, pp. 38-41 y June, 2012.

\section{How to cite this article:}

Lavhe, N. V., D. B. Undirwade, Nandini Gokte- Narkhedkar and Borkar, S. L. 2019. Locating Areas of High Density of Plant Parasitic Nematode, Rotylenchulus spp. in Vidarbha Region of Maharashtra State. Int.J.Curr.Microbiol.App.Sci. 8(12): 142-151. doi: https://doi.org/10.20546/ijcmas.2019.812.022 